\title{
Laser Guiding of Three Phase Tesla Coil High Voltage Discharges
}

\author{
SAMUEL KRISTIYANA, DILAN DWANURENDRA \\ Department of Electrical Engineering \\ Institute of Science \& Technology AKPRIND Yogyakarta \\ Kalisahak st. 28, Komplek Balapan, Yogyakarta \\ INDONESIA
}

\begin{abstract}
Guiding and triggering of discharges from a three phase Tesla coil type $280 \mathrm{kHz}$ AC high voltage source using filaments created by a femtosecond Terawatt laser pulse. Without the laser the discharges were maximum $30 \mathrm{~cm}$ long. With the laser straight, guided discharges up to $110 \mathrm{~cm}$ length were detected. The discharge length was limited by the voltage amplitude of the Tesla coil. A significant reduction of the breakdown voltage threshold due to the pre-ionization of the air gap by laser pulse filamentation was observed. The lifetime of filaments is measured by using time-resolved fluorescence spectrum, and the lifetime of filaments generated by dual fs laser pulses was doubled due to the re-ionization by the succeeding pulse.
\end{abstract}

Key-Words: - Tesla coil, discharge, high voltage,

Received: September 15, 2019. Revised: March 2, 2020. Accepted: March 31, 2020. Published: April 20, 2020.

\section{Introduction}

There have been several reports of the use of filaments created by high peak power femtosecond laser pulses for guiding high voltage (HV) discharges over meter length distances using unipolar pulsed and DC HV sources during recent years [1-6]. The work has mainly been motivated by triggering of lightning from storm clouds for e.g. airport protection. In our experiment we use a Tesla coil that is a low cost and compact source for pulsed $\mathrm{AC} \mathrm{HV}$ of a few hundred $\mathrm{kV}$. We do this to evaluate if triggering and guiding discharges from $\mathrm{AC} \mathrm{HV}$ sources over distances of several meters is possible. The discharge occurs during a time that is much shorter than the oscillation period of the AC voltage and the charge transfer is probably unidirectional. Possible applications exist in e.g. testing of discharge sensitivity of large electrical installations where it could be of interest to precisely direct multiple discharges to the same point.

A laser beam with peak power higher than the critical power, a few GW for $800 \mathrm{~nm}$ wavelength in air, will self-focus by the non-linear Kerr effect. When the intensity gets high enough a weak plasma is created by multi-photon ionization. A dynamic balance between the self-focusing and the defocusing from the plasma causes self-guiding of the laser beam over distances that may be order of magnitudes longer than the Rayleigh distance. This long thin structure is often called a filament. Detailed discussion of the filamentation process, its consequences and applications is available in e.g [79]. Often external focusing by lenses or mirror telescopes is used to control the position of the filament [10].

The rather weak plasma (of the order of 1016 $\mathrm{cm}-3$ [11]) that is generated by the laser beam will reduce the breakdown voltage of the air and guide HV discharges. The free electrons have a short lifetime, almost independently of the initial concentration the free electron density will be reduced to below $1011 \mathrm{~cm}-3$ in less than $1 \mu \mathrm{s}$ [12]. The removal of free electrons is to a large part caused by attachment of electrons to oxygen molecules, creating $\mathrm{O}_{2}$-ions. The electrons are less tightly bound to these ions than to the neutral molecules, reducing the necessary electrical field for impact ionization, and the effect of lowering the breakdown voltage may thus last longer than the lifetime of the free electrons. The exact process of the laser guided discharge is not yet well known and will not be clarified here even though we hope that the experimental data we provide can help in more detailed investigations. The mechanism behind triggering and guiding of discharges of DC voltage using ultrashort laser pulses is discussed in e.g [13$15]$.

\section{Three Phase Tesla Coil Discharge}

The Tesla coil HV source is an air core transformer developed by Nikola Tesla in 1891. 
There are today a number of design variations, we used a static spark gap variety.

The actual Tesla coil consists of two inductively resonantly coupled LC-circuits. The electrical setup is illustrated in Fig. 1. The primary circuit contains a large capacitance (CP) and a small inductance (LP), while the secondary circuit is a large inductance (LS ) and a top load toroid with a small capacitance to the ground (C S ). The primary circuit also includes a current switch in the form of a spark gap to induce a rapid change in the current. During the charging phase the spark gap is non-conducting and the primary capacitance is charged to $4 \mathrm{kV}$ from iron core transformers coupled to standard single phase $120 \mathrm{~V}, 60 \mathrm{~Hz}$ power. The power was provided by a petrol generator to avoid disturbances on the power grid of our facility. The charging circuit was modified to include a rectifier diode to keep the voltage over the capacitance constant once the peak voltage has been reached. The spark gap was set wide enough that no spontaneous discharge was possible from the charging voltage. To trigger the spark gap at a well-defined moment in time a nanosecond laser pulse from a Q- switched $\mathrm{Nd}$ :YAG laser was focused on one of the electrodes inside the spark gap. The laser pulse created a small plasma that initiated a full breakdown of the spark gap. When the primary LC-circuit is closed by the conducting plasma in the spark gap a rapidly (280 $\mathrm{kHz}$ in our case) oscillating current will transfer the stored energy back and forth between the capacitance and the inductance. By inductive coupling this current will generate an oscillating current in the secondary circuit where HV is created on the top load because the smaller capacitance needs higher voltage to store the same energy. The output HV waveform could not be directly measured, but from discharge measurements we infer that we generated a $280 \mathrm{kHz}$ oscillating voltage pulse with around $10 \mu$ s envelope. Two decoupling coils ( $\mathrm{L} \mathrm{C}$ ) are used to protect the charging circuit from the rapidly oscillating current. The primary capacitance was nominally $22 \mathrm{nF}$, giving a stored energy of $0.7 \mathrm{~J}$ per discharge. A metal screw was fixed to the Tesla coil top load to provide a break-out point for the discharges and concentrate them to the point where the laser beam passed.

\subsection{Laser}

The Terawatt \& Terahertz portable laser facility used for these experiments is a 4 TW Ti:Sapphire laser system delivering $160 \mathrm{~mJ}$ per pulse with a pulse duration of $40 \mathrm{fs}$. The central wavelength is $805 \mathrm{~nm}$ and the pulse repetition rate is $10 \mathrm{~Hz}$. The compact laser system sits on a $1.25 \mathrm{~m}$ by $2.5 \mathrm{~m}$ optical table. The laser system has been integrated into a standard sea container into which a modular clean room of class 100,000 was inserted. The clean room occupies about half of the container, the remaining part being used as a control room. The control room is filled with the air conditioning unit, the power supplies and chillers for the laser systems as well as the control rack. The user is able to fully control the laser either from the clean room, the control room or from the exterior of the container via an Ethernet link. The control room also acts as an airlock for the clean room.

In order to understand the characteristics of the triggering ability, we performed preliminary diagnostics of filaments. The length and the amount of free electrons of filaments produced by different pulse configurations is compared by sonography method [23,24]. In the plasma filaments, the air molecules are partially ionized and sound waves are formed. It has been found that the intensity of sound waves is related with the amount of free electrons in the plasma filaments. A microphone is placed perpendicularly to the filaments at a distance of 1 $\mathrm{cm}$ from the laser axis, as shown in Fig. 2. The acoustics signals are recorded by a digital oscilloscope after being amplified by an audio amplifier.

\subsection{Experimental method}

The freight container with the mobile laser system was inside a garage next to the test area to protect it from possible electromagnetic interference. The distance from the laser to the Tesla coil was around $15 \mathrm{~m}$. The laser beam was sent out through a pellicle-covered hole in the garage door. The beam was focused by a $5 \mathrm{~m}$ focal length lens (laser beam diameter of $2.5 \mathrm{~cm}$ FWHM) to control the position of the filaments. The FWHM length of the filamentfluorescence was measured to $0.8 \mathrm{~m}$, but there was clearly measurable fluorescence over 1.8 $\mathrm{m}$. The measurement of the UV fluorescence was performed by scanning a PMT covered by a UG11 filter along the laser beam path. The filament was positioned so that it started slightly before the HV electrode and the strongest part was approximately 1 $\mathrm{m}$ further away. 


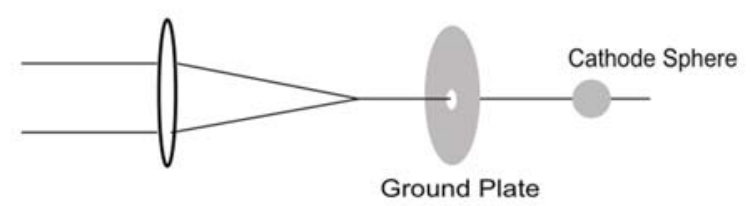

Fig. 1 Discharge setup

The laser passed right next to the two electrodes, with the metal tip on the Tesla coil closest to the laser and a metal target rod connected to ground that was used as second electrode further away, as shown in Fig. 2. The ground of the Tesla coil secondary circuit and the ground of the target rod were both connected to the same buried ground electrode. During all measurements care was taken that the filaments did not impact the electrodes and no plasma was generated on them. The filaments were however close enough to the electrodes that the weak outer part of the background photon bath hit the electrodes and the reflected laser light could be detected. The distance between the electrodes was varied from 10 to $150 \mathrm{~cm}$.

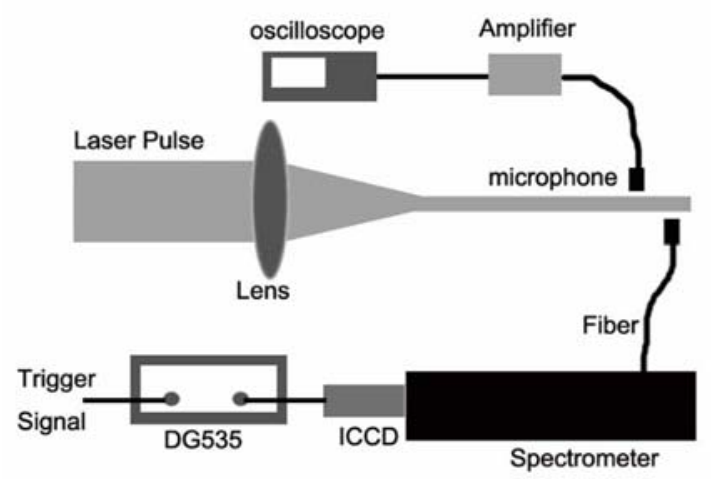

Fig. 2. The experiment setup for filaments diagnostics

\section{Experimental Result}

Using TW peak power ultrashort laser pulses to create a line of free charges in air through filamentation increases the maximum discharge distance from 30 to $110 \mathrm{~cm}$ in our experiment using a Tesla coil pulsed AC HV source. This is a larger effect from the laser guiding than what has been measured with pulsed DC HV sources where the reduction of the breakdown voltage for long discharges has been only around $30-40 \%[1,3]$.

The maximum guided discharge length achieved in these trials was $1.1 \mathrm{~m}$. The filament was strong past the target electrode and movement of the focusing lens by decimeters did not seem to impact the discharge probability, so the limiting factor is not the length and plasma distribution of the filaments formed. In pulsed $\mathrm{DC} \mathrm{HV}$ discharge experiments using 2.2 MV voltage discharge over up to $4.5 \mathrm{~m}$ gaps has been measured, but with a maximum guiding distance of $2.4 \mathrm{~m}$ and the remainder as unguided discharges [2]. There it was determined that the $1 \mu$ s lifetime of the laser initiation was the limiting factor for the guided length. As there is no evidence of unguided prolongation of the discharges in our case it is probable that the voltage is our main limitation.

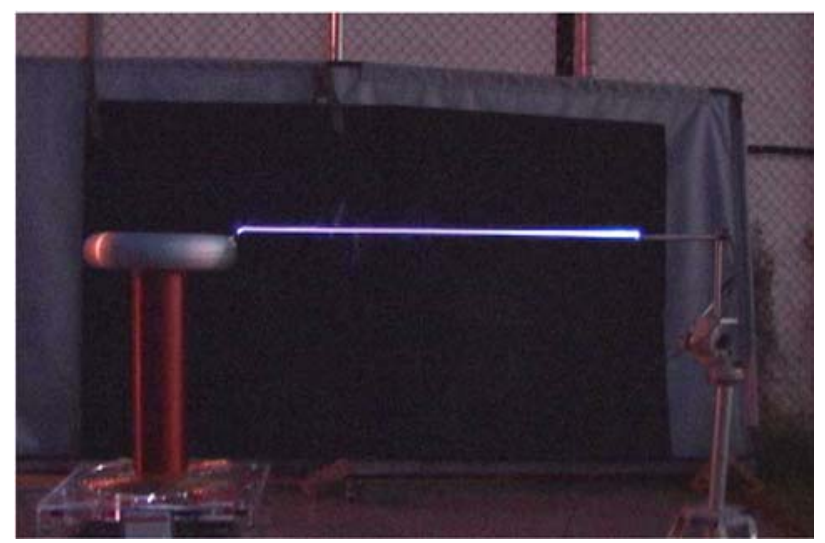

Fig. 3 The experiment setup with a $80 \mathrm{~cm}$ long guided discharge as imaged by video camera. The Tesla coil is seen on the left and the target electrode on the right.

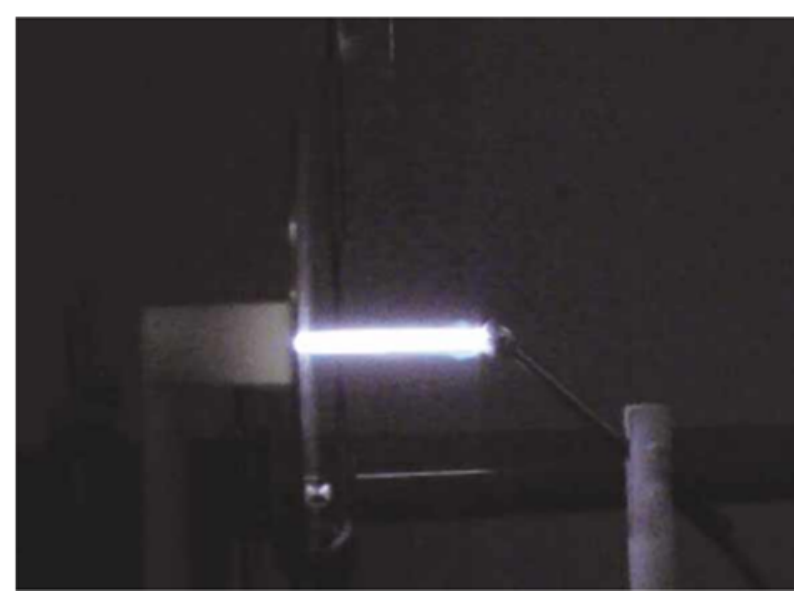

Fig. 4 The laser triggered discharge spark in a 45 mm gap.

With a pulsed DC HV-source it was concluded that the maximum guided distance is $2.4 \mathrm{~m}$ and that this distance is limited by temporal effects in the relation between the lifetime of the filament and the propagation speed of the discharge channel [2]. We are not able to use our data to determine if a similar limitation is present when using a pulsed AC HVsource and further measurements with higher 
voltage are needed to explore any temporal limitation. It is also possible that the frequency of the AC HV may have an impact on the maximum guiding distance. In our experiments we were not able to vary this parameter. Our observations were that the streamer propagation across the gap occurred as jumps when the instantaneous voltage was high. Further experiments are needed to determine if a high frequency with many short voltage peaks or a lower frequency with longer voltage peaks will produce the longest discharges.

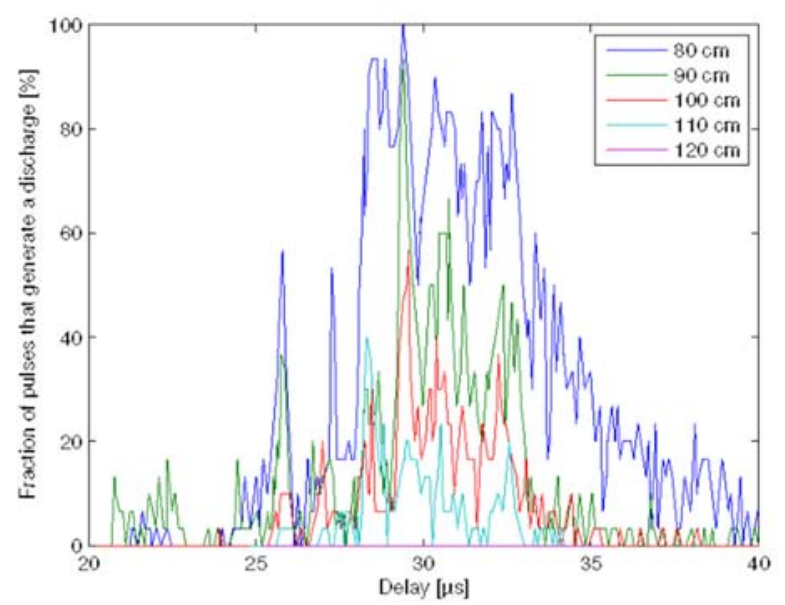

Fig. 5 Percentage of guided discharges measured at different gap distances for varying timing etween Tesla coil and laser pulses.

Without any filament bridging the electrode gap the discharges from the Tesla coil follow an erratic path as shown in Fig. 3. The maximum natural discharge length measured was $30 \mathrm{~cm}$. We were unable to probe the voltage with the available equipment without perturbing the HV circuit of the Tesla coil, and the peak voltage is thus unknown. The electric field necessary for breakdown is around $3 \times 106 \mathrm{~V} / \mathrm{m}$ for DC fields with electrodes that are large compared to the distance between them. The small radius electrodes used in our setup should reduce the necessary field strength. It is probable that the voltage from the Tesla coil was a few hundred $\mathrm{kV}$, while the theoretical value provided by the manufacturer for optimum tuning was $500 \mathrm{kV}$. The voltage may have varied during the experiments because of variation in temperature and moisture content of the air, which will affect the capacitance of the Tesla coil top electrode and thus the energy transfer efficiency from the primary to the secondary circuit.

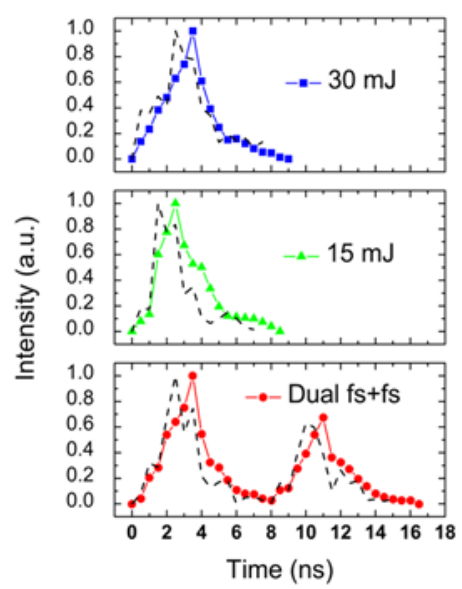

Fig. 6 The temporal evolution of the spectral line $383.8 \mathrm{~nm}$, the dashed line is the deconvolution results with the ICCD gate and delay time.

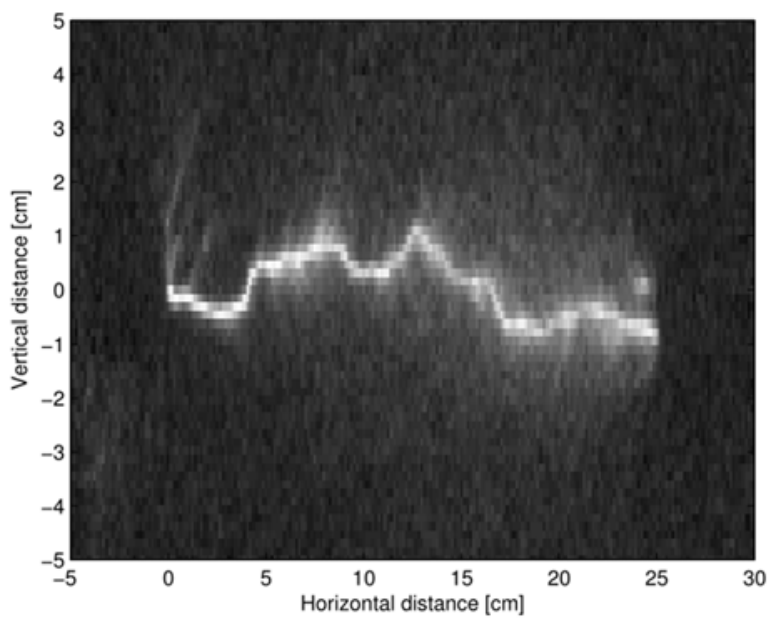

Fig.7 discharge across $25 \mathrm{~cm}$ gap without any filament.

When the gap between the electrodes was bridged by the laser-generated filament longer straight discharges occurred, as for the $100 \mathrm{~cm}$ gap in Fig. 4. At gaps longer than $80 \mathrm{~cm}$ initiation of a discharge did not always succeed, but most of the time a streamer that followed the laser path was generated even though it did not reach the target electrode. 


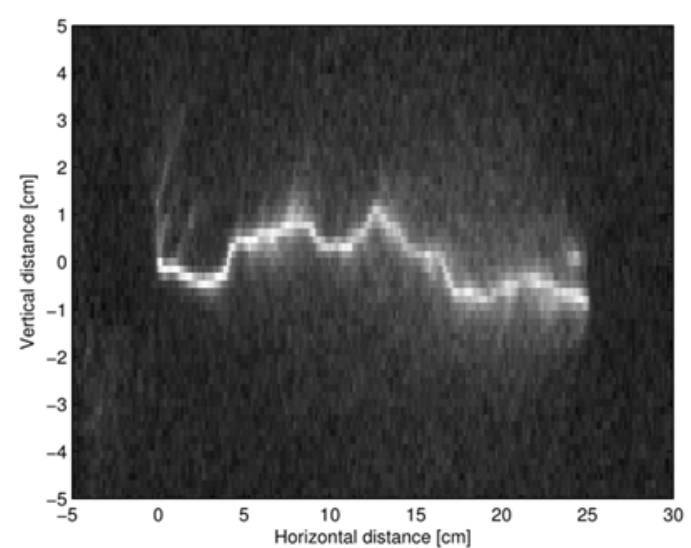

Fig. 8 ICCD camera image of guided discharge with $100 \mathrm{~cm}$ gap between the electrodes.

\section{Conclusion}

In conclusion we have shown triggering and guiding of electrical discharges over up to $1.1 \mathrm{~m}$ from a $280 \mathrm{kHz}$ AC high voltage Tesla coil using a filament created by self-focusing of a TW power femtosecond laser pulse. This is a factor 3.7 longer than the $30 \mathrm{~cm}$ discharges observed without the triggering laser. The streamers grew in leaps separated by half the oscillation period and the current-carrying discharge occurred as a sub-100 ns pulse when the leader bridged the gap. The guiding distance was limited by the voltage amplitude and further experiments with a higher voltage source is necessary to find the maximum guiding distance for discharges initiated by AC HV.

The triggering and guiding of the stationary HV discharges in 5-46 mm air gaps are demonstrated by using plasma filaments generated by single and dual fs laser pulse in air. The characteristics of filaments are studied firstly in terms of relative amount of free electrons and lifetime. The amount of free electrons in filaments is compared by using the sonography method. The maximum amount of free electrons is almost the same for the single $15 \mathrm{~mJ}$ pulse and the dual $15 \mathrm{~mJ}$ pulses, which is much lower than a single $30 \mathrm{~mJ}$ pulse. The lifetime of filaments is demonstrated by the time-resolved fluorescence spectrum, and the results have shown that the lifetime can be prolonged by adding a succeeding fs pulse. The lifetime of the plasma filaments generated by the single $15 \mathrm{~mJ}$ or $30 \mathrm{~mJ}$ pulses is about $5 \mathrm{~ns}$, and the lifetime of filaments can be prolonged by a $7 \mathrm{~ns}$ delayed succeeding fs pulse. The triggering and guiding ability of filaments is demonstrated by measuring the $\mathrm{HV}$ discharge length. The prolonging of discharge length up to 1.53 times of natural discharge length is demonstrated at $40 \mathrm{kV} \mathrm{HV}$ by using single $30 \mathrm{~mJ}$ pulse or the dual $15 \mathrm{~mJ} \mathrm{fs}+\mathrm{fs}$ pulses. The triggering ability of laser filaments can be improved by increasing the lifetime of the plasma filaments with dual fs + fs pulses. So the lifetime, consequently the triggering ability, is possible to be further increased by using a series fs pulses.

\section{References:}

[1] H. Li and Z. Jiang, On Automatic Resonant Frequency Tracking in LLC Series Resonant Converter Based on Zero-Current Duration Time of Secondary Diode, IEEE Transactions on Power Electronics, Vol. 31, No. 7, 2016, pp. 4956 - 4962.

[2] Zhe Zhang, Triggering and guiding HV discharge in air by filamentation of single and dual fs pulses, Optical Society of America, Vol. 17, No. 5, 2009, pp. 3461- 3468.

[3] R. Beiranvand, B. Rashidian, M. R. Zolghadri, et al, Using LLC resonant converter for designing wide-range voltage source, IEEE Transactions on Industrial Electronics., Vol. 58, No. 5, 2011, pp. 1746 - 1756.

[4] Hanqiu MO, Houjun Tang, A Magnetic Resonant Coupling Printed Spiral Coil System underDifferent Geometric Values, WSEAS Transactions On Circuits And System, Vol. 14, No. 1, 2015, pp. 442-447.

[5] Ali Moarrefzadeh, Study of workpiece thermal profile in Electrical Discharge Machining (EDM) process, WSEAS Transactions On Circuits And System, Vol. 7, No. 2, 2012, pp. 83-92.

[6] U. Kundu, K. Yenduri, and P. Sensarma, Accurate ZVS analysis for magnetic design and efficiency improvement of full-bridge LLC resonant converter, IEEE Transactions on Power Electronics, Vol. 32, No. 3, 2017, pp. 1703 - 1706.

[7] X. Fang, H. Hu, Z. J. Shen, et al, Operation mode analysis and peak gain approximation of LLC resonant converter, IEEE Transactions on Power Electronics, Vol. 27, No. 4, 2012, pp. $1985-1995$

[8] C. Akyel, S. I. Babic, S. Kincic and J. P. Lagacé, Magnetic Force Calculation of Some Circular Coaxial Coils in Air, Journal of Electromagnetic Waves and Applications, Vol. 21, No.9, 2007, pp. 1273-1283.

[9] M. K. Kazimierczuk and D. Czarkowski, Resonant Power Converters, John Wiley \& Sons, 2010.

[10] Babic S. and Akyel C., Mutual Inductance and Magnetic Force Calculations between Thick Coaxial Bitter Coil of Rectangular Cross Section with Inverse Radial Current and 
Filamentary Circular Coil with Constan Azimuthal Current, IET Electric Power Applications, Vol. 11, Issue 9, 2017, pp. 1596 1600.

[11] Hui-pin LIN, Xiao-guang JIN, Liang XIE, et al, A new variable-mode control strategy for LLC resonant converters operating in a wide input voltage range, Frontiers of Information Technology \& Electronic Engineering, Vol. 18, No.3, 2017, pp. 410-422. 